Cómo citar este trabajo: Talavera García, R., Valenzuela Montes, L. M., \& Soria Lara, J. A. (2018). Evaluando la influencia de la calidad de la distancia peatonal en la cobertura de paradas del metro ligero de Granada (España). Boletín de la Asociación de Geógrafos Españoles, 79, 2472, 1-25. http://dx.doi.org/10.21138/bage.2472

\title{
Evaluando la influencia de la calidad de la distancia peatonal en la cobertura de paradas del metro ligero de Granada (España)
}

Evaluating the influence of the quality of walking distance on the coverage of light rail stops in Granada (Spain)

\author{
Rubén Talavera García \\ rtalaverag@ugr.es \\ Luis Miguel Valenzuela Montes \\ lvmontes@ugr.es \\ Departamento de Urbanística y Ordenación del Territorio \\ Universidad de Granada (España) \\ Julio A. Soria Lara \\ julio.soria-lara@upm.es \\ Transport Research Centre (TRANSYT) \\ Universidad Politécnica de Madrid (España)
}

\section{Resumen}

Aunque las características del entorno urbano y social tienen una marcada influencia en la distancia peatonal al transporte público, existe una escasa atención a este fenómeno en el contexto de los análisis de accesibilidad. Sin embargo, considerar la calidad de la distancia peatonal en la evaluación de la accesibilidad podría constituir una herramienta útil para conseguir una mayor y mejor integración del transporte público en los entornos urbanos. En este artículo se diseña la herramienta Q-WD: Calidad de la Distancia Peatonal (Quality of Walking Distance) testeándola en el caso de la ciudad de Granada, y en concreto en varios entornos de movilidad vinculados a la línea 
de metro ligero. Los resultados muestran cómo la cobertura de las paradas se reduce en aquellos casos en los que la calidad peatonal de los entornos de parada es baja, mientras que los entornos de parada con alta calidad peatonal producen una extensión de la cobertura de parada del transporte público. Asimismo, los resultados de la aplicación de la herramienta Q-WD evidencian cómo el recuento total de población servida varía en los entornos peatonales cuando se considera la calidad de la distancia.

Palabras clave: accesibilidad; movilidad peatonal; diseño urbano; calidad; transporte público.

\begin{abstract}
The characteristics of the urban and social environment may have a strong influence in the walking distance to public transport. Nevertheless, scarce attention has been paid to this fact in the context of accessibility analysis. Even though, taking into account the quality of the walking distance in the evaluation of accessibility could be a useful tool to enhance higher public transport integration into urban environments. In this paper we develop the tool Q-WD: Quality of Walking Distance, which is tested in in the city of Granada, specifically on some mobility environments linked to stops of the light rail system. The results show how the catchment area decreases as the pedestrian environment quality diminishes, whilst in those pedestrian environments of high quality the catchment area expands. Furthermore, the results of applying the Q-WD tool allow to detect a change in the population served count, which is influenced by the distance quality.
\end{abstract}

Key words: accessibility; walking; urban design; quality; public transport.

\title{
1 Introducción
}

La movilidad peatonal, como base de la movilidad sostenible, es un factor clave para el éxito de la gestión urbana en términos económicos, sociales, ambientales y políticos (ARUP Group, 2016; Litman, 2016; Pozueta-Echavarri, Lamiquiz-Dauden \& Porto Schettino, 2009; van de Coevering \& Schwanen, 2006). Es además una pieza esencial para la intermodalidad con el sistema de transporte público debido a que son los desplazamientos a pie los que alimentan dicho transporte público, así como también son a pie los desplazamientos finales para acceder a la vivienda, los puestos de trabajo o las centralidades urbanas, entendiendo estas como lugares de confluencia con una marcada identidad. De ahí que la ubicación de la parada de transporte público condicione la cobertura, su nivel de servicio y su accesibilidad peatonal. A su vez, dichas medidas espaciales están condicionadas, en última instancia, por la distancia que el peatón recorre para alcanzar dicha parada o centralidad.

En este sentido la distancia peatonal no es una cuestión trivial y son numerosos los manuales que hacen referencia a ella, especialmente en la planificación orientada al transporte, también conocida 
como TOD's (Transit Oriented Development) en voz inglesa (Jacobson \& Forsyth, 2008). En ella se establece como distancia peatonal para la generación de coberturas y áreas de servicio distancias de entre 400 y 800 metros (1/4 y 1/2 milla) según el modo de transporte y destino (Curtis, Renne, \& Bertolini, 2009; Guerra, Cervero, \& Tischer, 2011; O’Sullivan y Morrall, 1996; Park, Deakin, \& Jang, 2013). No obstante, a pesar del consenso general a la hora de establecer una distancia peatonal a las paradas de transporte público o la generación de áreas de servicio a partir de dichas distancias, el concepto de distancia peatonal no es un concepto cerrado ni estático. Desde hace una década son varios los autores que analizan y discuten la necesidad de incluir medidas enfocadas a la mejora de la intermodalidad en el transporte, reforzando los desplazamientos a pie mediante el diseño urbano, como requisito para el éxito en la integración y gestión sostenible de los modos de transporte público en la ciudad (Cervero \& Kockelman, 1997; Hass-Klau \& Crampton, 2002; Olszewski \& Wibowo, 2005; Valenzuela Montes, Soria Lara, \& Talavera Garcia, 2011).

Desde este enfoque, y tomando como referencia la idea planteada por Gehl (1971) sobre la distancia peatonal aceptable como equilibrio entre la longitud del recorrido y su calidad; esta calidad estará determinada por el entorno social y por el entorno urbano, dado que para establecer una calidad es necesario tener en cuenta en qué medida el entorno urbano se adapta a las necesidades de la población. Por tanto, la distancia peatonal que la población está dispuesta a recorrer puede variar o matizarse en función de características demográficas (Hess, 2012) así como de características propias de diseño urbano (Ewing \& Cervero, 2010). Estas variaciones en la distancia peatonal sobre las que se evalúan las coberturas de paradas pueden dar lugar a que en ocasiones los niveles de servicio establecidos se vean sobrepasados por la realidad (Bhuyan y Nayak, 2013). A su vez, esta modificación se produce por la propia idiosincrasia del peatón, que le permite al mismo tiempo desplazarse y establecer interacciones tanto sociales como económicas (Venturi, Brown \& Izenour, 1977) con otros peatones (Gehl, 1971) y con el entorno (Jacobs, 1993).

El presente trabajo plantea como hipótesis; ¿es posible evaluar la calidad de la distancia peatonal y los efectos que ella conlleva respecto a la cobertura de parada? Para responder a esta pregunta se desarrolla una medida de la distancia peatonal al transporte público, basada en la calidad del entorno urbano y las características del entorno social. La medida se aplica en dos entornos de movilidad vinculados al corredor del metro ligero de Granada y que presentan características antagónicas, con el fin de evidenciar de manera clara el efecto que tanto el entorno urbano como social poseen en la accesibilidad a las paradas de un transporte público como el metro ligero.

Con esta finalidad se introduce a continuación el apartado de antecedentes, tras lo cual se detalla el método seguido para el desarrollo de la medida de distancia peatonal basada en la calidad. Posteriormente se muestran los resultados obtenidos de la aplicación del método en el contexto de dos entornos de movilidad de la ciudad de Granada, para en el apartado siguiente plantear la discusión de la repercusión que la incorporación de la calidad del entorno urbano y social puede 
dar lugar en el análisis de la cobertura del transporte público. Finalmente se presenta el apartado de conclusiones.

\section{Antecedentes}

Respecto a la propia medida de distancia empleada en los artículos revisados, éstos muestran la frecuente utilización de unas distancias preestablecidas de un cuarto de milla o de media milla (400 y 800 metros respectivamente). La distancia de 400 metros se asocia a modos más locales como el autobús y el metro ligero, mientras que la distancia de 800 metros se asocia, según la revisión efectuada a modos con mayores distancias entre paradas como el metro, el BRT o el tren. El uso de una distancia preestablecida puede ser de utilidad para comparar casos de estudio (Guerra et al., 2011); para analizar un factor asociado a la distancia pero sin que se analice en sí la repercusión sobre la propia distancia (Hess \& Almeida, 2007); o como solución en aquellos casos en los que la información existente no permita una evaluación de la influencia de los factores sobre la distancia. En este sentido, Guerra, Cervero y Tischer (2011) muestran que la distancia peatonal a la parada de transporte público no tiene verdadera relevancia, sus análisis están efectuados únicamente teniendo en cuenta el número de viajeros en función de la distancia a parada. Dejando a un lado las distancias preestablecidas, en los casos en los que se analiza la distancia mediante encuesta o cálculos las distancias peatonales muestran una alta variabilidad, aun cuando dicha distancia se relaciona con el mismo modo de transporte. Este hecho tiene su explicación en la propia variabilidad de las características del entorno y de la población, o en otros factores de diversa índole que se vinculan a la movilidad peatonal (Valenzuela-Montes y Talavera-García, 2015) y por consiguiente a la distancia peatonal. En esta línea se encuentran trabajos como los de El-Geneidy, Grimsrud, Wasfi, Tetreault y Surprenant-Legault (2014), Larsen y El-Geneidy (2010) y Seneviratne (1985) en Canadá u O'Sullivan y Morrall (1996) en Estados Unidos, en los cuales se obtienen distancias peatonales influenciadas por diferentes factores y que difieren de la distancia peatonal estándar que sugieren las autoridades y los manuales TOD.

En cuanto al análisis llevado a cabo en aquellos casos en los que se obtiene la distancia peatonal mediante una encuesta a la población, esta muestra una mayor asociación de factores ligados al concepto de la distancia peatonal. Como se puede apreciar en la selección de artículos que analizan la cobertura de parada (Tabla 1), en la mayoría de los casos se analizan factores demográficos como edad o género como muestran artículos como el de García-Palomares, Gutiérrez y Cardozo (2013), en el cual la distancia peatonal a las paradas de metro en Madrid, varía en función de los distintos grupos de población existente. También se encuentran frecuentes alusiones a la raza y a los ingresos (B. B. Brown y Werner, 2009; Park, Deakin, y Jang, 2013). Por otra parte, y en base a los trabajos internacionales analizados, es frecuente la asociación de la distancia con factores relativos al entorno urbano como la propia tipología de entorno (Seneviratne, 
1985), el diseño del mismo (El-Geneidy et al., 2014; O’Sullivan \& Morrall, 1996) o los usos del suelo (Aultman-Hall, Roorda, \& Baetz, 1997; Moniruzzaman, Páez, Paez, \& Páez, 2012). Estos factores pueden hacer que la distancia peatonal varíe, dando lugar, como sugieren Park, Deakin, y Jang (2013), a que en los lugares con una alta calidad del entorno la distancia se incremente, mientras que por el contrario, lugares con una baja calidad del entorno, la distancia peatonal decrezca. Esta relación entre distancia peatonal y factores considerados varía en función del modo de transporte que se considere. Así, el metro ligero muestra una media de factores por artículo más alta (5 factores por artículo) siendo la tipología urbana, población y sexo y edad de la población los factores más frecuentes. Le siguen el autobús y el BRT con 4 factores por artículo y cuyos factores más frecuentes son la población y valor de la propiedad respectivamente, aunque en este último caso el número de artículos puede condicionar dichos resultados. En otras palabras y atendiendo al número de factores considerados, el metro ligero tiene un mayor potencial para la integración, por ser un modo local con una notable interacción con el entorno urbano y la población que en ellos residen. En contraposición, modos de transporte público como el metro y el tren se sitúan a la cola en cuanto a consideración de factores por artículos lo que pone en evidencia que son modos de transporte con poca capacidad de integración, por sus propias condiciones estructurales respecto al entorno urbano.

Si bien, a la vista de la revisión elaborada, es evidente la asociación entre factores y distancia peatonal, son escasas las referencias en las que se tratan de integrar todos los factores en un método que pueda evaluar la accesibilidad a las paradas de transporte público. A este respecto, cabe destacar el trabajo elaborado por Olszewski y Wibowo (2005) en el que utilizan una distancia peatonal equivalente a partir de la cual evalúan la accesibilidad peatonal a las estaciones de transporte público. No obstante, si bien el trabajo realizado supone un claro avance en las medidas de accesibilidad, su enfoque se centra en los factores como obstáculos que originan un incremento en la distancia (respecto a la distancia geométrica) necesaria para acceder a las paradas de transporte. Es por tanto pertinente considerar que el trabajo desarrollado por estos autores puede aplicarse también en sentido positivo, o en otras palabras, que la calidad de los factores presentes en los entornos urbanos pueden dar lugar a una distancia relativa menor que la distancia de la red, dando lugar a una mayor accesibilidad a las paradas de transporte público.

En este contexto, considerar la influencia de diversos factores del entorno urbano y social en la distancia peatonal al transporte público parece una vía lógica de mejorar las medidas de análisis de la accesibilidad y avanzar en la integración espacial del transporte público. No obstante, esta mejora no está carente de complejidad. Es por ello fundamental conservar aquellos rasgos de las medidas de accesibilidad que han sido un éxito, como son por ejemplo los niveles de servicio, ya que permiten una fácil comprensión y aplicación en la planificación y en la toma de decisiones. Del mismo modo, la simplicidad y facilidad de análisis en las medidas basadas en la distancia deben 
seguir estando presente. Sin embargo es necesario pasar de la consideración de una distancia preestablecida a una distancia aceptable, en la que se tenga en cuenta las características propias de la ruta (Gehl, 1971). Además, sería necesario que, manteniendo la facilidad de entendimiento y aplicación, se incorporasen las necesidades de acceso y preferencias respecto al entorno urbano de la población, como sucede en las medidas de accesibilidad basadas en la utilidad.

Tabla 1. Revisión de artículos que analizan la distancia peatonal

\begin{tabular}{|c|c|c|c|c|}
\hline Autores & Medida & Modo & $\begin{array}{l}\text { Dist. } \\
(\mathrm{m})\end{array}$ & Factores \\
\hline (Aultman-Hall et al., 1997) & $\mathrm{F}$ & bus & 300 & Población, comercios, colegios \\
\hline $\begin{array}{l}\text { (J. Brown, Thompson, } \\
\text { Bhattacharya \& Jaroszynski, } \\
\text { 2014) }\end{array}$ & - & bus & - & $\begin{array}{l}\text { Población, trabajo, raza, ingresos, edad y sexo, } \\
\text { propósito, vehículo }\end{array}$ \\
\hline (Chen et al., 2010) & $\mathrm{F}$ & bus & 800 & $\begin{array}{l}\text { Población, edad y sexo, centros de salud, } \\
\text { distribución y frecuencia del transporte, } \\
\text { educación }\end{array}$ \\
\hline (Das, Maitra, \& Boltze, 2012) & $\mathrm{F}$ & bus & 1000 & precios \\
\hline (Delmelle, Li \& Murray, 2012) & $\mathrm{F}$ & bus & 400 & - \\
\hline $\begin{array}{l}\text { (Djurhuus, Sten Hansen, } \\
\text { Aadahl, \& Glümer, 2016) }\end{array}$ & $\mathrm{F}$ & general & 1000 & Población, tipología urbana \\
\hline (Donaldson, 2006) & $\mathrm{F}$ & tren & 400 & Población, trabajo \\
\hline (El-Geneidy et al., 2014) & E & $\begin{array}{l}\text { bus } \\
\text { tren }\end{array}$ & $\begin{array}{c}524 \\
1259\end{array}$ & $\begin{array}{l}\text { Población, ingresos, diseño urbano, edad y } \\
\text { sexo, momento del día, propósito, vehículo }\end{array}$ \\
\hline (García-Palomares et al., 2013) & $\mathrm{E}$ & metro & 800 & Población, nacionalidad, edad y sexo, vehículo \\
\hline (Hernández \& Witter, 2015) & E & $\begin{array}{l}\text { bus } \\
\text { metro }\end{array}$ & $\begin{array}{c}776 \\
1100\end{array}$ & 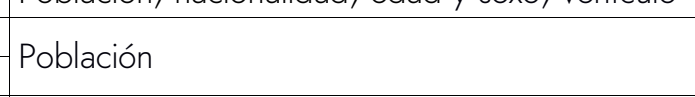 \\
\hline (Hess, 2012) & E & bus & 400 & $\begin{array}{l}\text { Raza, ingresos, diseño urbano, tipología urbana, } \\
\text { edad y sexo, vehículo }\end{array}$ \\
\hline (Hess \& Almeida, 2007) & $\mathrm{F}$ & metro ligero & 400 & Valor de la propiedad \\
\hline (Kim, 2011) & $\mathrm{F}$ & general & 800 & $\begin{array}{l}\text { Tiendas, parques, restaurantes, raza, ingresos, } \\
\text { tipología urbana, edad y sexo, inquilinos, } \\
\text { discapacidad, educación }\end{array}$ \\
\hline (Koushki, 1988) & $\mathrm{E}$ & Bus & 536 & \\
\hline $\begin{array}{l}\text { (Kuby, Barranda \& Upchurch, } \\
\text { 2004) }\end{array}$ & $\mathrm{F}$ & metro ligero & 800 & Población, trabajo, inquilinos, distancia. \\
\hline (Mitchell et al., 2003) & $\mathrm{F}$ & bus & 500 & $\begin{array}{l}\text { Tiendas, parque, confort, restaurantes, ingresos, } \\
\text { tipología urbana, edad y sexo, discapacidad, } \\
\text { centro de salud }\end{array}$ \\
\hline $\begin{array}{l}\text { (Morency, Trepanier \& Demers, } \\
\text { 2011) }\end{array}$ & C & $\begin{array}{l}\text { bus } \\
\text { tren }\end{array}$ & $\begin{array}{r}152 \\
950\end{array}$ & \multirow{4}{*}{$\begin{array}{l}\text { Tiendas, parque, restaurante, edad y sexo, } \\
\text { colegio, propósito } \\
\text { Población, trabajo, ingresos, edad y sexo, valor } \\
\text { propiedad, tamaño casa, seguridad } \\
\text { Población, trabajo, comercios, restaurantes, } \\
\text { tipología urbana, edad y sexo, hora del día, } \\
\text { frecuencia transporte, propósito, aparcamiento, } \\
\text { ubicación }\end{array}$} \\
\hline (Pivo \& Fisher, 2011) & $\mathrm{F}$ & bus & 800 & \\
\hline \multirow[b]{2}{*}{ (Seneviratne, 1985) } & \multirow[b]{2}{*}{$\mathrm{E}$} & bus & 250 & \\
\hline & & metro ligero & 287 & \\
\hline $\begin{array}{l}\text { C: distancia calculada } \\
\text { F: distancia fijada o preestable } \\
\text { S: distancia encuestada }\end{array}$ & & & & \\
\hline
\end{tabular}

Fuente: elaboración propia

Sobre ello, son varios los autores que han avanzado en la medida de una distancia peatonal aceptable al transporte público en base a una encuesta a la población, como el trabajo de El- 
Geneidy et al. (2014) o mediante la inclusión de factores del entorno urbano y social como Park, Deakin y Jang, (2013). Sin embargo, a pesar del avance que estos trabajos suponen, la consideración de un gran número de factores en la composición de los índices da lugar que resulte costosa su aplicación por cuestión de tiempo y disponibilidad de datos. Desde la perspectiva planteada, este trabajo pretende seguir avanzando en la evaluación del desarrollo de una distancia peatonal basada en la calidad del entorno urbano y en las características de la población, manteniendo una facilidad de entendimiento y aplicabilidad que permita utilizarse en la toma de decisiones.

Antes de desarrollar la metodología es necesario tener en cuenta el concepto de entorno de movilidad y el concepto de entorno peatonal. En primer lugar, se definen los entornos de movilidad como aquellos en el que las personas pueden llegar (nodo) y desarrollar diversas actividades (lugar) (Bertolini, 1999), estando dicho entorno determinado por el conjunto de condiciones externas que podrían tener influencia en la presencia de personas en un determinado lugar (Bertolini \& Dijst, 2003). En segundo lugar, los entornos de movilidad peatonal o entornos peatonales se definen como los espacios dominados por los desplazamientos a pie, donde otros modos, incluyendo vehículos a motor, pueden tener lugar, pero en el que los peatones tienen claramente la prioridad de movimiento (Zacharias, 2001). Por tanto, para que pueda darse esta prioridad, no sólo es necesario una gestión de la movilidad orientada hacia modos no motorizados, sino que es además necesaria un entorno de calidad que propicie y fomente los desplazamientos a pie. Así, en este trabajo, el entorno peatonal supone una espacio de prioridad para el peatón sustentado en la calidad tanto a nivel estructural, como a nivel de usos del suelo y de diseño urbano, posibilitando el fomento de este tipo de movilidad no motorizada y la vinculación y acceso al transporte público.

\section{Metodología}

La metodología propuesta se fundamenta en la idea de calidad peatonal de Talavera-Garcia, SoriaLara y Valenzuela-Montes (2014) aplicada a la distancia a la parada de transporte público. Así, la calidad de una determinada calle estará determinada por su capacidad para satisfacer las necesidades de un peatón (accesibilidad, seguridad, confort y atractivo) a la hora de caminar. Esta calidad da lugar a una menor percepción de la distancia dando lugar a una mayor accesibilidad por parte de los usuarios.

La calidad de la distancia peatonal (Q-WD por el acrónimo en inglés de quality of walking distance) es una medida de accesibilidad que se aplica en tres fases.

1) Se evalúa la calidad peatonal de los entornos de movilidad a partir de indicadores, umbrales de calidad, y ponderación según la percepción de la población 
2) Se incorpora los valores de calidad peatonal como atributo a la red de calles sobre la que se va a llevar a cabo el análisis de redes (mediante el módulo NetworkAnalyst de ArcGIS 10) en base a la calidad de la distancia peatonal, y se realizan las transformaciones necesarias para dicha finalidad

3) Se generan coberturas de parada (mediante Service Areas del módulo NetworkAnalyst que se basa en el algoritmo de Dijkstra) basadas en la calidad de la distancia peatonal que permite la evaluación de otras cuestiones como la población servida.

Por otra parte, la medida Q-WD está diseñada en base al siguiente método que se estructura en varias etapas.

\subsection{Etapas del método}

El método para la evaluación de la accesibilidad a las paradas de metro ligero basado en la calidad de la distancia peatonal toma como referente el trabajo realizado por Olszewski y Wibowo (2005). Estos autores diseñan un método de evaluación de la distancia peatonal en el entorno de las paradas de transporte teniendo en cuenta el efecto que las características de esos entornos tienen sobre la accesibilidad. Sin embargo, el diseño del método que desarrollan los autores, llamado Equivalent Walking Distance, se basa en el efecto negativo que las características del entorno tienen en la accesibilidad, al incrementar la distancia equivalente a las paradas respecto a la distancia real (medida en la red). En contraposición, el método que se desarrolla a continuación considera el efecto de la calidad (ya sea en términos positivos o negativos) en la distancia peatonal a las paradas de metro ligero.

\section{Figura 1. Resumen gráfico}

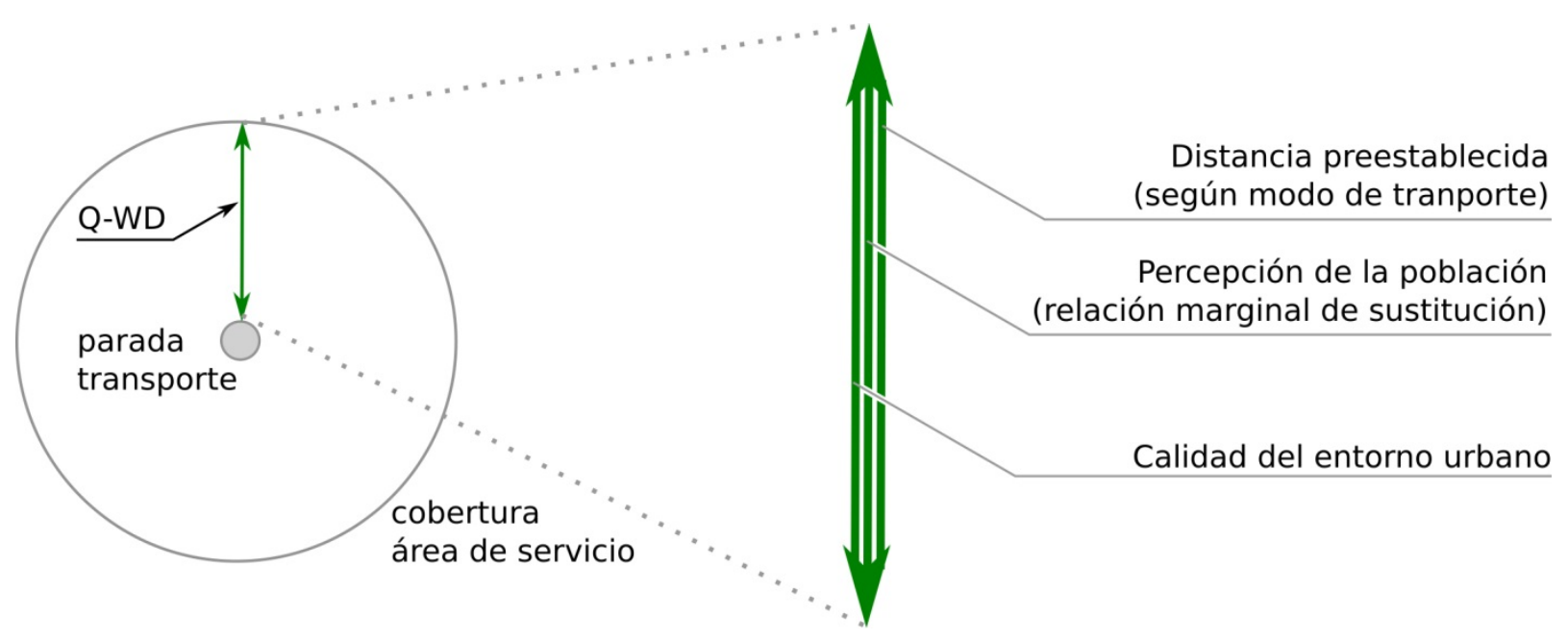

Fuente: elaboración propia 
El método se divide en tres etapas. La primera relativa a la distancia peatonal a la parada según el modo de transporte. Una segunda etapa en la cual se determina la calidad peatonal de los entornos de movilidad o de parada de transporte público, teniendo en cuenta las características del entorno urbano y la percepción que de ellas pose la población. Y la última etapa relativa a la agregación de componentes para la obtención de la distancia peatonal basada en la calidad (Q-WD).

\section{Distancia preestablecida según modo de transporte}

Para obtener la distancia peatonal basada en la calidad es necesario tener como punto de partida la distancia estándar a considerar en función del modo de transporte. Estas distancias suelen ser de entre 400 y 600 metros para modos de transporte más locales como autobuses, tranvía, metros ligeros y autobús rápido (Agència d’Ecologia Urbana de Barcelona, 2010; The city of Calgary, 2004; Wright \& Hook, 2010), y distancias de entre 800 y 1200 metros para modos de transporte con una mayor velocidad y distancia como metro y tren (Vuchic, 2005).

Si bien atendiendo a la bibliografía la distancia que con mayor frecuencia se utiliza respecto al metro ligero se establece entre 400 y 800 metros, en esta investigación se ha escogido como distancia preestablecida la distancia de 500 metros. Esta distancia se justifica en base al ámbito de testeo y modo de transporte seleccionado para la aplicación del método que se desarrolla. En este sentido el proyecto de la línea 1 de metro ligero de Granada establece una distancia peatonal de 500 metros para la evaluación de la cobertura de las paradas (Ferrocarriles de la Junta de Andalucía, 2008) (Ver datos generales del proyecto en el epígrafe Ámbito de testeo).

\section{Calidad peatonal}

Para evaluar el efecto del entorno urbano en la distancia peatonal al transporte público se utilizan los resultados sobre la calidad peatonal obtenidos por Talavera-Garcia \& Soria-Lara (2015), a partir de los factores presentes en la Tabla 2. Cada uno de los factores hace referencia a los necesidades que se deben satisfacer para que tenga lugar la acción de caminar: accesibilidad, seguridad, confort y atractivo (Alfonzo, 2005). La selección de estos factores (Tabla 2) responde a criterios de relevancia, aplicabilidad y comprensión (Niemeijer \& de Groot, 2008, p. 18; Talavera-Garcia \& Soria-Lara, 2015). Los criterios de relevancia y compresión son criterios frecuentes respecto a la dimensión de gestión, mientras que el criterio de aplicabilidad forma parte de la dimensión práctica. Además se tiene en cuenta el criterio de umbrales, el cual resulta de gran utilidad al permitir determinar cuándo llevar a cabo una acción (Niemeijer \& de Groot, 2008, p. 18). 
Tabla 2. Resumen de los factores e indicadores

\begin{tabular}{|l|l|l|l|l|}
\hline Necesidad & Factores & Indicadores & Unidades & Comentarios \\
\hline Accesibilidad & Conectividad & Conectividad & \# intersecciones & $\begin{array}{l}\text { Número de intersecciones por } \\
\text { segmento de calle }\end{array}$ \\
\cline { 2 - 5 } & $\begin{array}{l}\text { Anchura } \\
\text { acera }\end{array}$ & $\begin{array}{l}\text { Anchura } \\
\text { acera }\end{array}$ & metros & $\begin{array}{l}\text { Lémite de velocidad de la vía. A } \\
\text { Seguridad }\end{array}$ \\
$\begin{array}{l}\text { Velocidad } \\
\text { tráfico } \\
\text { el número de carriles }\end{array}$
\end{tabular}

Fuente: Talavera-Garcia y Soria-Lara (2015)

Para obtener los valores de calidad para cada uno de los factores considerados, los resultados de los indicadores se estandarizan siguiendo diferentes criterios, tanto recomendaciones en aquellos casos en los que existan o en base a la distribución de los valores resultantes, tal y como exponen Talavera-Garcia y Soria-Lara (2015). Esta estandarización de los resultados para cada uno de los factores estaría en una escala de 1 a 5 en la que 5 representa la máxima calidad. Además de los umbrales, para cada uno de los factores evaluados, es necesario conocer la importancia que le da la población a cada uno de los factores a la hora de caminar, hecho que permite evidenciar la percepción del entorno urbano que posee la población y sus necesidades. Utilizando los valores obtenidos por Talavera-Garcia y Soria-Lara (2015) mediante una encuesta online a la población residente de Granada en el que se evaluaba la actitud y preferencias en el diseño urbano de la calle a la hora de caminar, se analiza la relación marginal de sustitución de los valores de importancia que la población otorga a los diferentes factores respecto a la distancia. Este coeficiente permite homogeneizar e introducir la percepción de la población respecto a los factores en la fórmula de la calidad de la distancia peatonal (Q-WD).

\section{Calidad de la distancia peatonal}

Una vez obtenido los valores de calidad, es necesario realizar un proceso de agregación de la información para poder incorporarlo en el análisis de redes. En este sentido, se opta por un diseño de red viaria con división cada dos metros, que permita extraer la información de la calidad obtenida con gran detalle. Así, una vez introducidos los valores de calidad como atributo para el análisis de redes, dicho atributo de la calidad del tramo de calle se convierte en un valor de peso que modulará la distancia preestablecida (WD) en función de la calidad, con la finalidad de obtener una distancia peatonal basada en la calidad peatonal (Q-WD). 
Una vez obtenidos los valores de los factores y sus correspondientes pesos, se introducen en la siguiente fórmula para obtener el valor de calidad peatonal (Q-WD).

$$
(Q W D)=W D\left(\left(\sum W_{n} Q_{n}\right) / n\right)
$$

QWD = Calidad de la distancia peatonal

WD $=$ Distancia peatonal

W = Peso o coeficiente de relación marginal de sustitución

$Q=$ Calidad del factor considerado

$\mathrm{n}=$ número de factores considerados

Esta ponderación de la distancia en base a la calidad se realiza siguiendo la Tabla 3, en la que se puede apreciar que el valor de calidad 3 tiene un efecto neutro sobre la distancia, mientras que valores inferiores o superiores, disminuyen o incrementan, respectivamente, la distancia peatonal. Para ello, se toma como referencia la distancia máxima (800 metros) recogida en la revisión de artículos referentes a la distancia peatonal al metro ligero (Talavera-Garcia \& Valenzuela-Montes, en prensa). Con la distancia máxima y la distancia peatonal dada por el proyecto se establece la ponderación, de tal manera que cuando la calidad es óptima (5) la distancia peatonal se incrementa hasta el máximo recogido en la literatura, mientras que por el contrario si la calidad es mínima, la distancia disminuiría hasta la distancia peatonal mínima.

Tabla 3. Efecto de los niveles de calidad peatonal en la medida de distancia

\begin{tabular}{|c|c|}
\hline Calidad & Ponderación \\
\hline 1 & 0,4 \\
\hline 2 & 0,7 \\
\hline 3 & 1 \\
\hline 4 & 1,3 \\
\hline 5 & 1,6 \\
\hline
\end{tabular}

Fuente: elaboración propia

\section{2 Ámbito de testeo}

Cómo ámbito de testeo para aplicar los indicadores contenidos utilizados en la calidad de la distancia peatonal (Q-WD) se han seleccionado cuatro paradas de la línea de metro ligero de Granada. La línea 1 de metro ligero (Tabla 4) conecta los núcleos metropolitanos de Albolote, Maracena y Armilla con la ciudad de Granada con un trazado de aproximadamente 16 kilómetros, a lo largo de los cuales se disponen un total de 26 estaciones de metro (23 en superficie y 3 subterráneas). El diseño del trazado responde a la demanda de acceso a diferentes centralidades 
urbana y metropolitanas como son la estación de autobuses, hospital Virgen de las Nieves, estación de ferrocarriles, campus centro de la Universidad de Granada, Palacio de Deportes, campo de fútbol Nuevo Los Cármenes y Campus Tecnológico de la Salud.

\section{Tabla 4. Cuadro resumen metro ligero granada "metropolitano"}

\begin{tabular}{|l|c|}
\hline$N^{\circ}$ de líneas & 1 \\
\hline Longitud total $(\mathrm{m})$ & 15923 \\
\hline $\mathrm{N}^{\circ}$ de estaciones & 26 \\
\hline $\mathrm{N} .^{\circ}$ de estaciones soterradas & 3 \\
\hline Estimación de viajeros (mill/año) & 12.9 \\
\hline Pob. servida & 138248 \\
\hline Duración & $45^{\prime}$ \\
\hline Fase & Puesta en marcha \\
\hline
\end{tabular}

Fuente: elaboración propia a partir de Consejería de Obras Públicas

y Transportes Junta de Andalucía (2017)

De forma más específica, se han seleccionado para el testeo cuatro paradas de metro ligero, en el contexto de dos entornos de movilidad (Figura 2), un entorno de movilidad motorizada y otro entorno de movilidad local descrito por Soria-Lara (2011). Estos entornos de movilidad seleccionados ligados al corredor de metro ligero de Granada, que se encuentra en fase de pruebas, suponen dos entornos de movilidad con características antagónicas como se detalla a continuación.

En primer lugar y como expone Soria-Lara, Valenzuela-Montes y Pinho (2015), el entorno de movilidad orientado al tráfico se caracteriza por un flujo de vehículos privados muy elevado y una movilidad de proximidad irrelevante. Además es un entorno predominantemente residencial con una densidad de menos de 3000 casas $/ \mathrm{km}^{2}$, donde la población no puede satisfacer sus necesidades diarias debido a la baja actividad económica (53,56 usos/1000 casas), lo que justifica su alto volumen de tráfico (384,27 vehículos/uso). En segundo lugar, el entorno de movilidad local posee una gran autonomía, siendo poca la movilidad que recibe desde otras secciones censales. Además, este entorno posee una alta densidad de población (>9500 casas $/ \mathrm{km}^{2}$ ), donde la población residente puede cubrir sus necesidades diarias. Respecto al mix en el modo de transporte, el modo más extendido en estos lugares es a pie, siendo bajos los índices de motorización tanto de vehículos privados (46,02 vehículos/uso) como de transporte público (4.3 autobuses/uso del suelo).

Estos entornos de movilidad poseen características diametralmente opuestas que permiten evaluar cómo la distancia peatonal a las estaciones de metro ligero se ve condicionada por la calidad del entorno peatonal que presentan los diferentes entornos de movilidad, y la percepción que posee la 
población respecto a los factores más importantes del entorno urbano a la hora de emprender la acción de caminar.

\section{Figura 2. Ámbito de testeo}

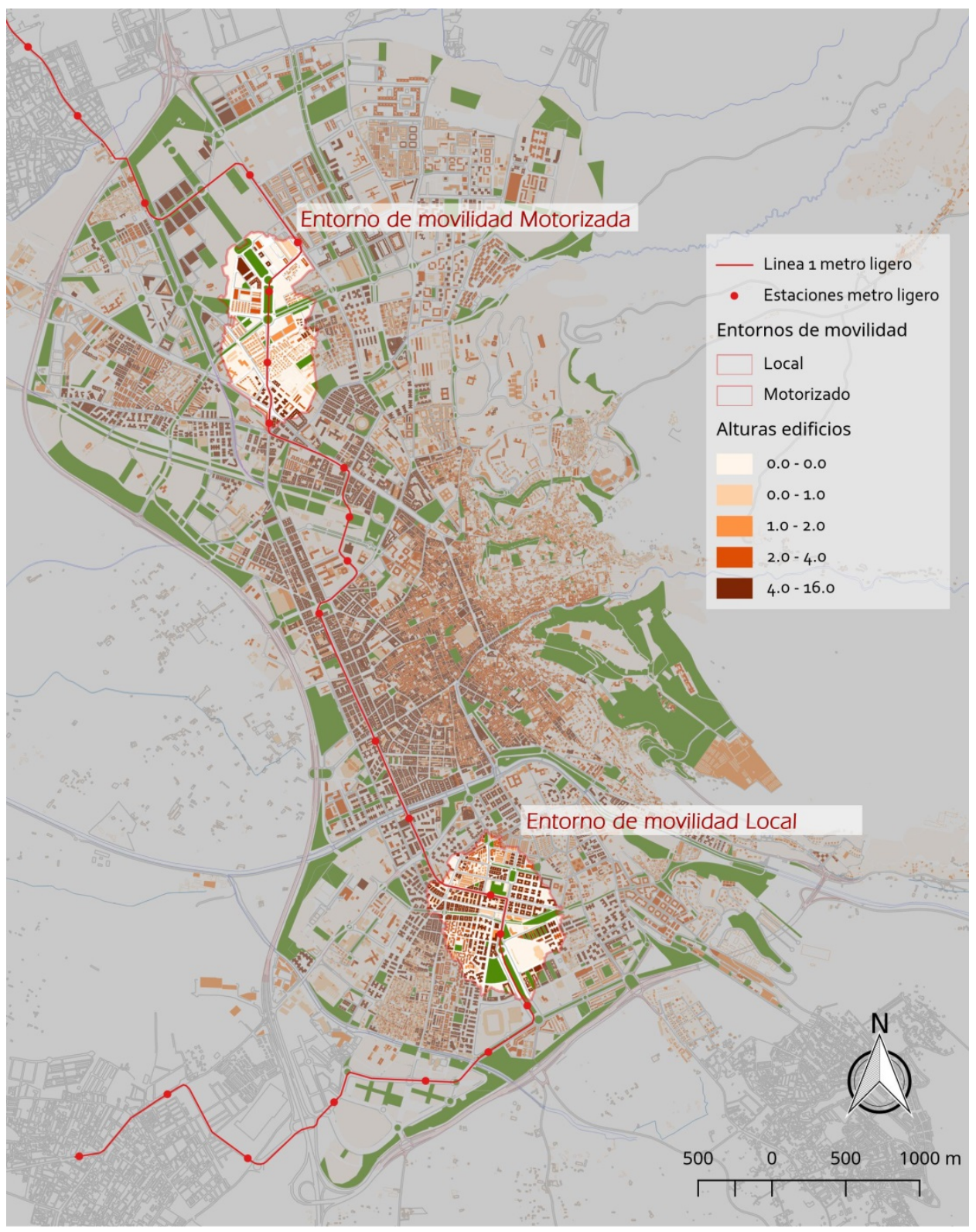

Fuente: elaboración propia

\section{Resultados y discusión}

Los resultados obtenidos de la aplicación de la fórmula Q-WD se muestran en dos partes, una primera parte relativa a los resultados de los indicadores de calidad peatonal de los entornos de movilidad evaluados y una segunda parte relativa a la medida de accesibilidad basada en cobertura para cada uno de los entornos evaluados en función de la calidad. 


\section{IV.1. Calidad peatonal de los entornos de movilidad}

Los resultados de la calidad de los entornos de movilidad evaluados (Figura 3 y Tabla 5), muestran cómo existen diferencias sustanciales entre el entorno de movilidad motorizada y el entorno de movilidad local. Mientras que en el entorno de movilidad local las calles presentan una calidad alta, las calles del entorno de movilidad motorizada presentan una calidad peatonal media lo que las convierte en menos confortables y atractivas para ser recorridas por los peatones a la hora de acceder a las estaciones de metro ligero.

Figura 3. Calidad peatonal de los entornos de movilidad

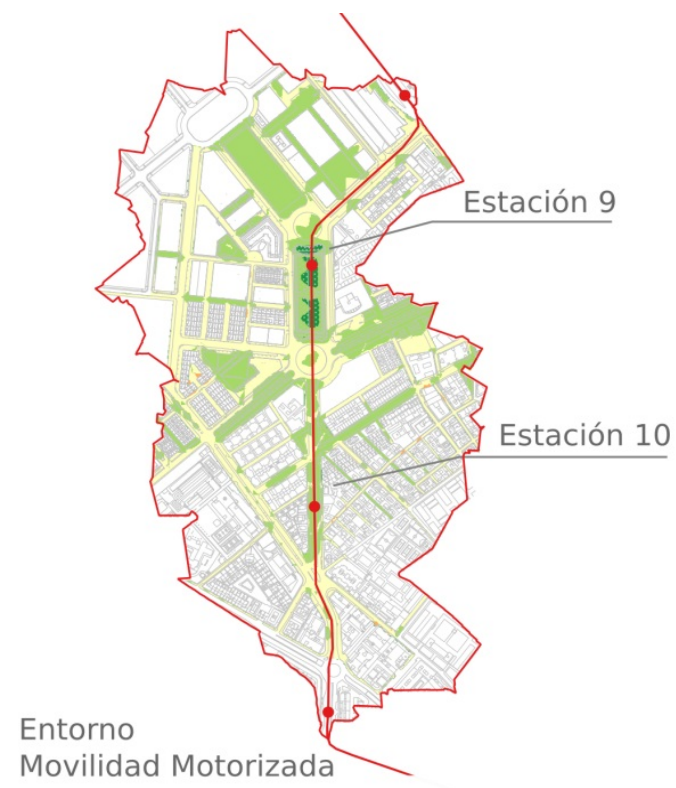

Movilidad Motorizada

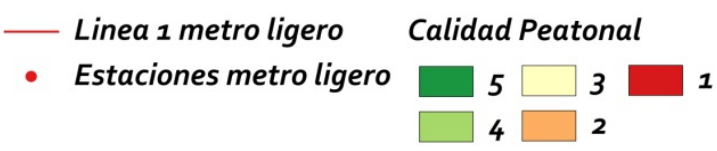

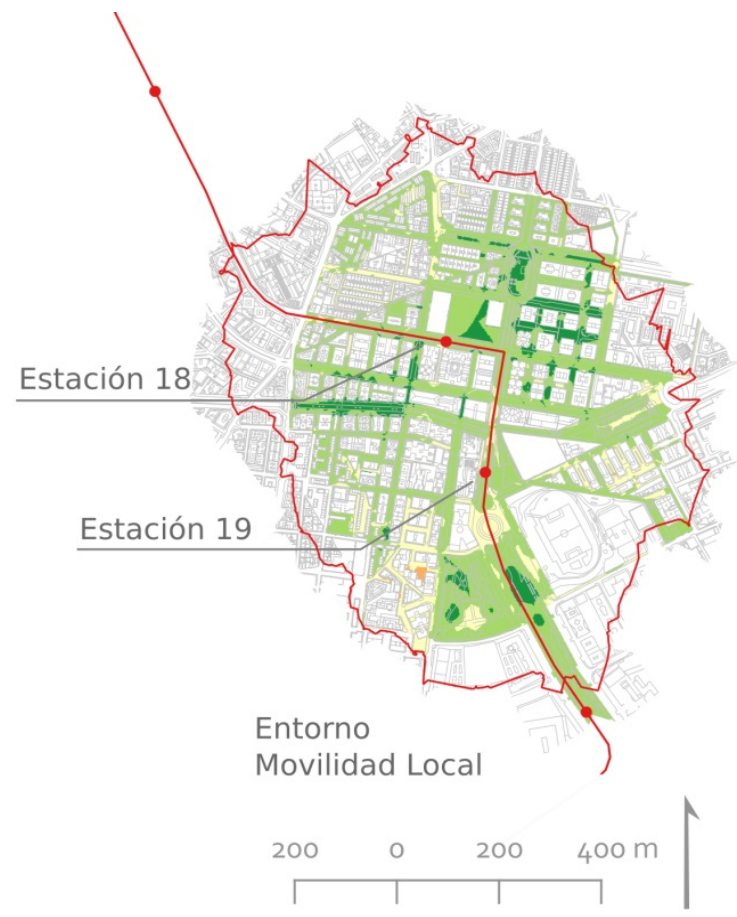

Fuente: elaboración propia a partir de Talavera-Garcia y Soria-Lara (2015)

Tabla 5. Valores sintéticos de la calidad peatonal de los entornos de movilidad

\begin{tabular}{|l|c|c|c|c|c|c|}
\cline { 2 - 7 } \multicolumn{1}{c|}{} & \multicolumn{5}{c|}{$\begin{array}{c}\text { Calidad de las calles medido en } \\
\text { \% de superficie respecto al total }\end{array}$} & $\begin{array}{c}\text { Calidad } \\
\text { media }\end{array}$ \\
\cline { 2 - 7 } & 5 & 4 & 3 & 2 & 1 & \\
\hline $\begin{array}{l}\text { Entorno de } \\
\text { movilidad } \\
\text { motorizada }\end{array}$ & $2.13 \%$ & $35.87 \%$ & $60.04 \%$ & $1.96 \%$ & $0 \%$ & 3 \\
\hline $\begin{array}{l}\text { Entorno de } \\
\text { movilidad local }\end{array}$ & $4.42 \%$ & $69.15 \%$ & $26.17 \%$ & $0.27 \%$ & $0 \%$ & 4 \\
\hline
\end{tabular}

Fuente: elaboración propia a partir de Talavera-Garcia y Soria-Lara (2015) 
Estos valores de calidad, por tanto, muestran como el entorno de movilidad local presenta una mejor calidad respecto al entorno urbano que el entorno de movilidad motorizado. Este hecho da lugar a que el entorno de movilidad local favorezca que la población recorra una mayor distancia para acceder a la parada de metro ligero. A este hecho contribuye, entrando en detalle, los espacios públicos vinculados, las calles peatonales y la presencia de bulevares, ya que por sus características, son lugares en los que destaca su accesibilidad dentro de la trama urbana; la seguridad por ser lugares en los que la presencia de vehículos motorizados es nula o residual; el confort, por estar asociados con la presencia de arbolado y vegetación lineal; y finalmente por ser lugares atractivos, en los que hay una fuerte socialización, motivo por el cual suele estar rodeado de comercios. No obstante, en el caso del entorno de movilidad motorizada la presencia de los citados tipos de espacios se concentra en vías principales, dando lugar a que la calidad del entorno disminuya considerablemente en las calles traseras o más distanciadas del trazado del metro ligero. Serían, por tanto, necesarias políticas que potenciasen la movilidad no motorizada a través de la mejora del entorno urbano y la diversidad de usos, de tal manera que favoreciese la accesibilidad a las paradas de metro ligero.

\subsection{Cobertura de parada basada en la calidad de la distancia peatonal}

Una vez obtenidos los valores de calidad para la movilidad peatonal de las calles que componen los entornos de movilidad e introducidos los valores en la red peatonal, se obtienen las coberturas de las paradas de metro ligero de acuerdo a la calidad de la distancia peatonal. En este sentido y comparando con la cobertura estándar basada en la distancia se puede apreciar como en el entorno de movilidad motorizada, al poseer sus calles menor calidad peatonal, da lugar a una cobertura de paradas menor a la cobertura obtenida con la distancia preestablecida. Por el contrario, en el entorno de movilidad local se observa que la cobertura de las estaciones de metro ligero aumenta en la distancia dada la mayor calidad de sus calles.

En concreto, tomando como medida base los 500 metros expuestos en el proyecto de metro ligero, la distancia peatonal en el entorno de movilidad motorizada aumentaría de media, en base a la calidad peatonal de sus calles, 58 metros hasta una distancia de 558,43 metros, mientras que en

el entorno de movilidad local la distancia peatonal incrementaría de media próxima a los 75 metros alcanzando por tanto los 574,40 metros.

En este contexto, los resultados obtenidos evidencian que un entorno de movilidad peatonal, entendiendo este como un entorno local que potencia la peatonalidad (walkability), permite generar unas distancias peatonales de acceso al transporte público mayores que un entorno de movilidad motorizada con un diseño urbano poco orientado al peatón. 


\section{Figura 4. Cobertura basada en distancia estándar y calidad de la distancia peatonal}
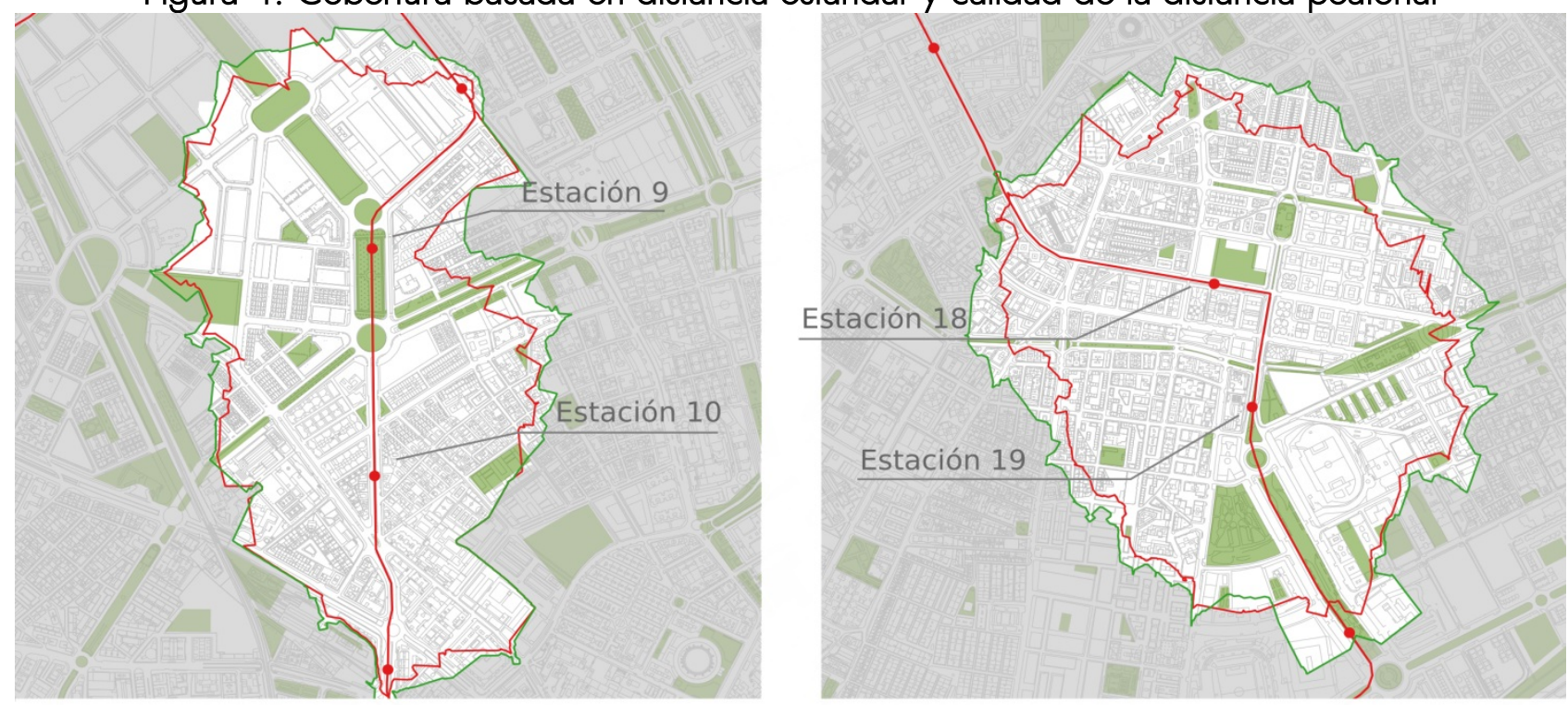

- Línea 1 metro ligero

Área de servicio estandar

- Estaciones metro ligero $\Sigma$ Área de servicio Q-WD

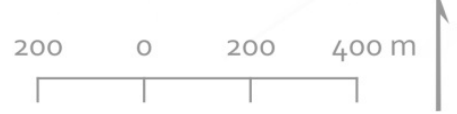

Fuente: elaboración propia

Estos resultados en los que se aumenta la distancia peatonal al transporte público cuando se incrementa la calidad del recorrido se encuentra en la línea del trabajo llevadas a cabo por ElGeneidy, Grimsrud, Wasfi, Tetreault y Surprenant-Legault (2014) según el cual la distancia peatonal a los autobuses aumentaría en función de las características propias hasta los 524 metros. En el trabajo desarrollado en Canadá por Larsen y El-Geneidy (2010) la distancia peatonal por motivos de trabajo aumentaba hasta los 650 metros; en el de Seneviratne (1985) la distancia a metro ligero resultó en 287 metros. Por otra parte, en el contexto de Estados Unidos, O'Sullivan y Morrall (1996) obtienen distancias peatonales medias de 444 metros y 527 metros (considerando el percentil 75 de las observaciones) para la estación de Sunnyside. Estos valores difieren de otras estaciones analizadas por su carácter local y por su vinculación a diferentes características del aparcamiento y puestos de trabajo. En esta línea, cabe destacar el trabajo de Park et al. (2013) en el que prueban como, en base a datos obtenidos mediante encuestas y generando modelos a partir de las características del entorno urbano y social, la calidad del entorno de las estaciones permite aumentar la distancia peatonal al transporte público sobre todo a lo largo del corredor de transporte.

\subsection{Implicaciones de la cobertura de parada basadas en la calidad de la distancia peatonal}

Más allá del aumento o disminución de la distancia peatonal y la generación de las coberturas de parada, estas variaciones en función de la calidad de la distancia peatonal tiene notables implicaciones sobre la población servida (Figura 5). En este sentido, dada la baja densidad de 
población en el entorno de movilidad motorizada, la población servida varía de manera menos intensa de la que tienen lugar en el entorno de movilidad local, donde la densidad de población es muy alta.

Figura 5. Comparativa cobertura de las paradas, solapamientos e implicaciones en población
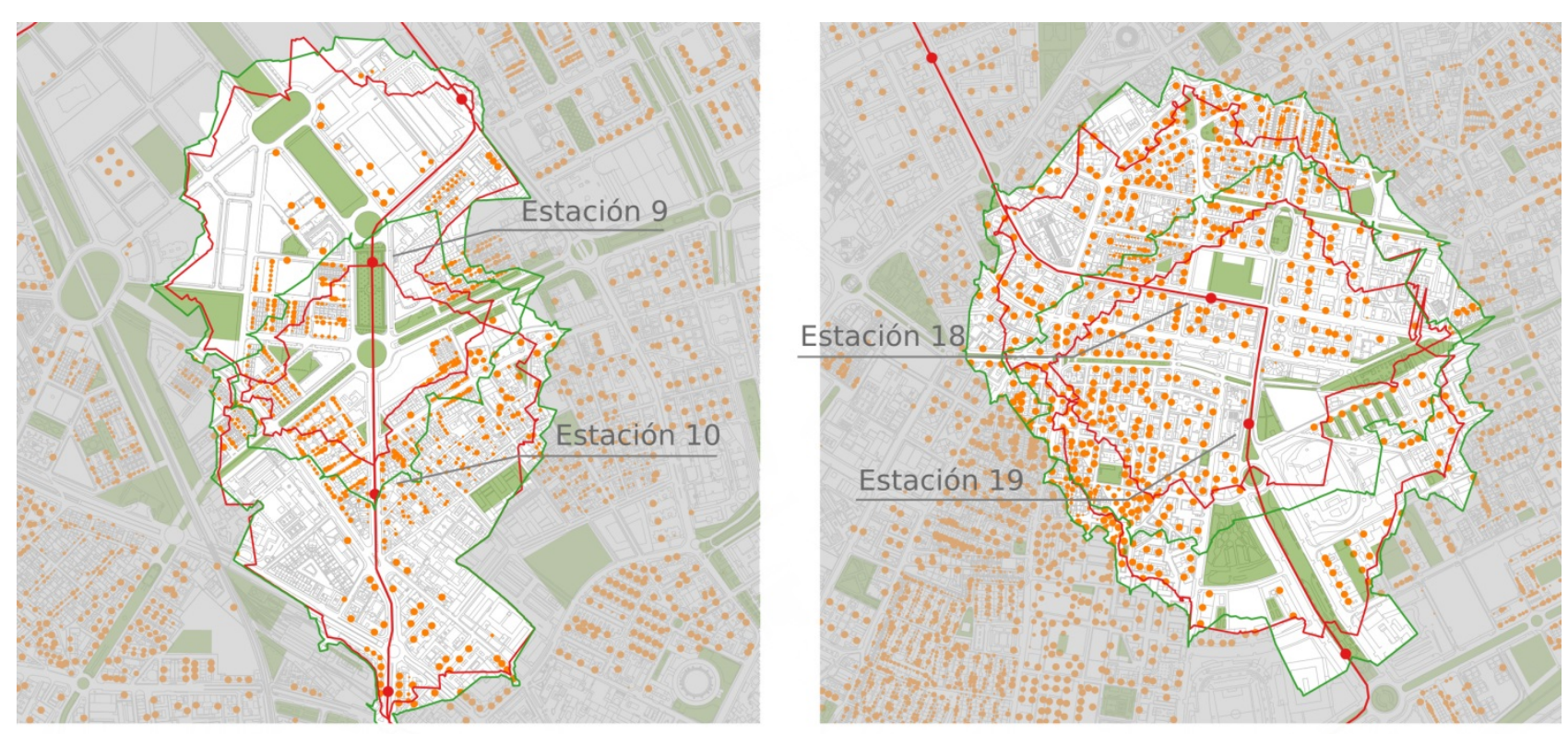

- Linea 1 metro ligero Población acceso vivienda

- Estaciones metro ligero . Q1

$\bigcirc$ Área de servicio estandar. $Q_{2}$

$\sum$ Área de servicio $Q-W D \cdot Q_{3}$

- $\mathrm{O}_{4}$

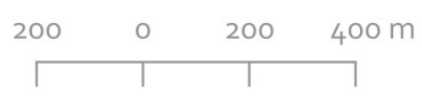

Fuente: elaboración propia

Profundizando en esta cuestión, el estudio de demanda de la red de metro ligero (Ferrocarriles de la Junta de Andalucía, 2008) establece para las paradas 18 y 19 (lo que corresponde al entorno de movilidad local) una población servida de 23307 (23 157 mediante nuestro análisis de la cobertura de parada). No obstante, la consideración de una cobertura a partir de la calidad de la distancia peatonal supone una disminución de la población considerada del 11,9\% en el entorno de movilidad motorizada, mientras que en el entorno de movilidad local habría un aumento del 21,3\% de población servida respecto a la población bajo cobertura medida con la distancia establecida por el proyecto de metro ligero (Figura 6). Además de estos cambios en la población servida, cabe prestar atención al solapamiento que se producen en las paradas que se encuentran en los entornos de movilidad evaluados. Así pues, el cambio de medida de la distancia peatonal da lugar, en el caso del entorno de movilidad motorizada, a un incremento de 666 residentes en la zona de solapamiento, mientras que por el contrario, en el entorno de movilidad peatonal tiene lugar un incremento de 7204 personas que residen en la zona de solapamiento de las coberturas de parada. Este incremento de la cobertura de la parada 18, así como el solapamiento entre 
coberturas de las paradas de metro, supone que la población residente servida por la parada 18 represente el 88,45\% del total de la población servida en el entorno de movilidad local, lo que da lugar a cuestionarse la correcta ubicación de la parada 19 de la línea de metro ligero.

Figura 6. Comparativa de la población servida en los entornos de movilidad motorizada y local según el tipo de distancia peatonal

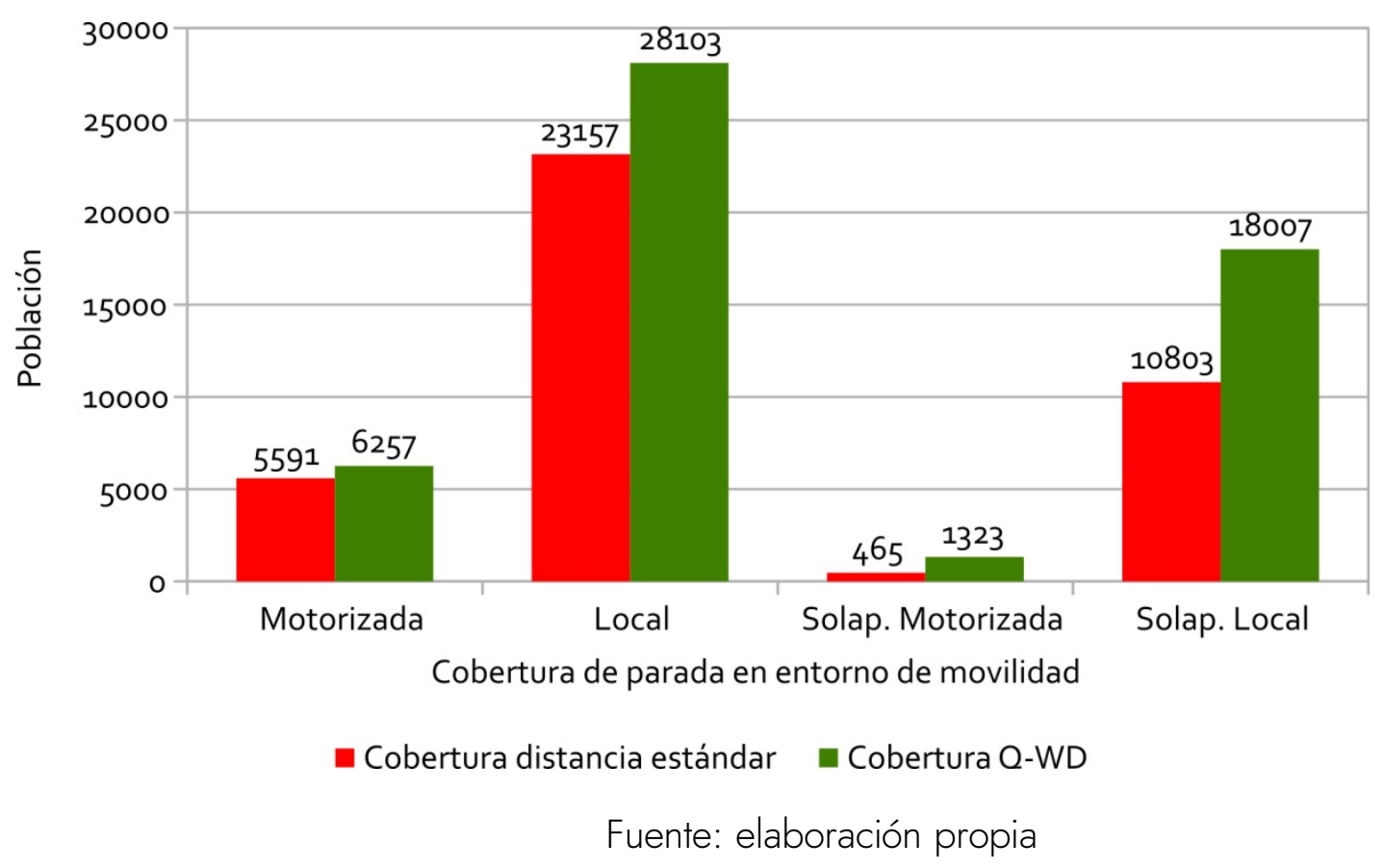

\section{Conclusiones y líneas futuras}

De acuerdo con los resultados obtenidos en la aplicación de la calidad de la distancia peatonal (QWD) y comparando con la medida basada en una distancia peatonal estándar al transporte público, el método desarrollado en este trabajo permite identificar de forma detallada el efecto del entorno urbano en el acceso al transporte público, en base a las necesidades de la población respecto de caminar. En este sentido, los entornos de movilidad local poseen características más favorables que los entornos de movilidad motorizada para que tenga lugar una movilidad peatonal y accesibilidad al transporte público. En otras palabras, el entorno de movilidad local presenta las características necesarias para ser considerado un entorno peatonal es decir, un entorno en el que la movilidad peatonal es prioritaria dada las características sociales y un diseño favorable que cubre las necesidades de la población respecto de caminar o acceder al transporte público, en este caso.

Se ha comprobado (Tabla 6) cómo la evaluación de la accesibilidad en base a la distancia euclidiana da lugar a una importante sobreestimación de la distancia respecto a la distancia medida en la red, hecho que es más evidente en aquellos entornos con una configuración espacial más intrincada (mayor factor de desvío) como sucede en el entorno de movilidad motorizado. Por otra parte, la evaluación basada en la calidad de la distancia (Q-WD) da lugar a que la población 
pueda recorrer una mayor distancia dada la percepción que poseen respecto a la calidad de los entornos. En este sentido, las paradas del entorno de movilidad local muestran factores de calidad ligeramente más elevados que los correspondientes para el entorno de movilidad motorizada.

Tabla 6. Valores sintéticos de la calidad peatonal de los entornos de movilidad

\begin{tabular}{|c|c|c|c|c|c|c|}
\hline $\begin{array}{l}\text { Entorno } \\
\text { movilidad }\end{array}$ & Estación & $\begin{array}{l}\text { Distancia } \\
\text { proyecto }\end{array}$ & $\begin{array}{l}\text { Distancia media } \\
\text { en la red }\end{array}$ & $\begin{array}{c}\text { Distancia media } \\
\text { Q-WD }\end{array}$ & $\begin{array}{l}\text { Factor de } \\
\text { desvío }^{1}\end{array}$ & $\begin{array}{l}\text { Factor de } \\
\text { calidad }^{2}\end{array}$ \\
\hline \multirow{2}{*}{ Motorizada } & Estación 9 & 500 & 692,06 & 567,02 & 1,38 & 1,13 \\
\hline & Estación 10 & 500 & 707,81 & 549,83 & 1,42 & 1,10 \\
\hline \multirow{2}{*}{ Local } & Estación 18 & 500 & 620,26 & 569,01 & 1,24 & 1,14 \\
\hline & Estación 19 & 500 & 632,05 & 579,78 & 1,26 & 1,16 \\
\hline
\end{tabular}

Fuente: elaboración propia

El diseño del método para la medida de la distancia peatonal basada en la calidad tiene importantes mejoras respecto a los métodos tradicionales basados en una distancia peatonal preestablecida. La primera es que el método desarrollado tiene en consideración, a través de los factores utilizados, las distintas necesidades respecto a caminar, lo que da lugar a una medida más ajustada a las características de la movilidad peatonal. La segunda es que la calidad de la distancia peatonal se ha diseñado teniendo presente los factores más relevantes en la movilidad peatonal según su frecuencia en la bibliografía académica. La tercera mejora respecto a la medida tradicional es la incorporación de las preferencias de la población respecto al diseño urbano desde su punto de vista como peatones.

Además de estas ventajas es necesario destacar la flexibilidad y facilidad de uso del método desarrollado. Por ejemplo, no precisa de grandes conocimientos estadísticos para entender los resultados. Todo ello se basa en la idea de desarrollo como una caja blanca que hace el método adaptable a las necesidades propias de evaluación de la accesibilidad al transporte público y las características del lugar en el que se aplica, mediante la adición o modificación de los valores de ponderación de los factores.

Finalmente, la calidad de la distancia peatonal al transporte público supone un método con gran aplicabilidad en el proceso de toma de decisiones en el ámbito de la gestión urbana sostenible y particularmente de la movilidad sostenible, dado que se mantiene el nivel de aplicabilidad de la medida tradicionalmente usada de la distancia peatonal para la generación de coberturas. Además el método Q-WD integra cuestiones del diseño urbano que son fundamentales tanto para una buena integración espacial del transporte público en la ciudad, como para diferentes centralidades urbanas que son atractoras de desplazamientos susceptibles de ser realizados a pie. 
Respecto a las líneas de avance, se contempla la posibilidad, una vez esté en funcionamiento la línea de metro ligero, de contrastar a través de una encuesta los resultados obtenidos. Esta encuesta a la población usuaria del metro ligero permitiría calibrar y validar el método desarrollado. Así mismo, se plantea como avance, la aplicación del método desarrollado a diferentes centralidades o propósitos.

Agradecimientos: Este trabajo ha sido posible gracias a la financiación de la Junta de Andalucía y los fondos FEDER para el Proyecto de Excelencia P12-RNM-1514: "Instrumentos para la valoración de escenarios urbanos frente al cambio climático. Diseño de un software para la evaluación ambiental (MITIGA)" (2014-2018).

Declaración responsable: Las/os autoras/es declaran que no existe ningún conflicto de interés en relación a la publicación de este artículo. Rubén Talavera-García diseñó, desarrolló y aplicó el método. Realizó la busqueda de antecedentes y analizó los resultados obtenidos. Luis M. Valenzuela-Montes contribuyó en el diseño del método propuesto, así como en el desarrollo de la introducción y la discusión. Julio A. Soria-Lara contribuyó con los datos de partida, aspectos metodológicos y la discusión. 


\section{Bibliografía}

Agència d'ecologia Urbana de Barcelona. (2010). Sistema de indicadores y condicionantes para ciudades grandes $y$ medianas. Retrieved from http://www.ecourbano.es/imag/pdf/INDICADORES CIUDADES GRANDES Y MEDIANAS.pdf

Alfonzo, M. (2005). To Walk or Not to Walk? The Hierarchy of Walking Needs. Environment and Behavior, 37(6), 808-836. https://doi.org/10.1177/0013916504274016

Arup Group (2016). Cities Alive. Towards a walking world. London. Reetrieved from https://www.arup.com/-/media/arup/files/publications/c/cities-alive_towards-a-walkingworld_lowres.pdf

Aultman-Hall, L., Roorda, M., \& Baetz, B. W. (1997). Using GIS for evaluation of neighborhood pedestrian accessibility. Journal of Urban Planning and Development, 123(1), 1017. https://doi.org/10.1061/(ASCE)0733-9488(1997)123:1(10)

Bertolini, L. (1999). Spatial Development Patterns and Public Transport: The Application of an Analytical Model in the Netherlands. Planning Practice and Research, 14(2), 199210. https://doi.org/10.1080/02697459915724

Bertolini, L., \& Dijst, M. (2003). Mobility Environments and Network Cities. Journal of Urban Design, 8(1), 27-43. https://doi.org/10.1080/1357480032000064755

Bhuyan, P. K., \& Nayak, M. S. (2013). A Review on Level of Service Analysis of Urban Streets. Transport Reviews, 33(2), 219-238. doi: https://doi.org/10.1080/01441647.2013.779617

Brown, B. B., \& Werner, C. M. (2009). Before and After a New Light Rail Stop: Resident Attitudes, Travel Behavior, and Obesity. Journal of the American Planning Association, 75(1), 512. https://doi.org/10.1080/01944360802458013

Brown, J., Thompson, G., Bhattacharya, T., \& Jaroszynski, M. (2014). Understanding Transit Ridership Demand for the Multidestination, Multimodal Transit Network in Atlanta, Georgia: Lessons for Increasing Rail Transit Choice Ridership while Maintaining Transit Dependent Bus Ridership. Urban Studies, 51(5), 938-958. https://doi.org/10.1177/0042098013493021

Cervero, R., \& Kockelman, K. (1997). Travel demand and the 3Ds: Density, diversity, and design. Transportation Research Part D: Transport and Environment, 2(3), 199-219. https: //doi.org/10.1016/S1361-9209(97)00009-6

Chen, J., Shaw, S.-L., Yu, H., Lu, F., Chai, Y., \& Jia, Q. (2011). Exploratory data analysis of activity diary data: a space-time GIS approach. Journal of Transport Geography, 19(3), 394404. https://doi.org/10.1016/i.jtrangeo.2010.11.002 
Curtis, C., Renne, J. L., \& Bertolini, L. (2009). Transit oriented development: making it happen. Farnham: Ashgate Publishing.

Das, S. S., Maitra, B., \& Boltze, M. (2012). Planning of Fixed-Route Fixed-Schedule Feeder Service to Bus Stops in Rural India. Journal of Transportation Engineering-Asce, 138(10), 12741281. https://doi.org/10.1061/(ASCE)TE.1943-5436.0000419

Delmelle, E. M., Li, S., \& Murray, A. T. (2012). Identifying bus stop redundancy: A gis-based spatial optimization approach. Computers Environment and Urban Systems, 36(5), 445455. hitps://doi.org/10.1016/i.compenvurbsys.2012.01.002

Djurhuus, S., Sten Hansen, H., Aadahl, M., \& Glümer, C. (2016). Building a multimodal network and determining individual accessibility by public transportation. Environment and Planning $B$ : Planning and Design, 43(1). hitps://doi.org/10.1177/0265813515602594

Donaldson, R. (2006). Mass rapid rail development in South Africa's metropolitan core: Towards a new urban form? Land Use Policy, 238(3). hitps://doi.org/10.1016/j.landusepol.2005.02.003

El-Geneidy, A., Grimsrud, M., Wasfi, R., Tétreault, P., Surprenant-Legault, J., Tetreault, P., \& Surprenant-Legault, J. (2014). New evidence on walking distances to transit stops: identifying redundancies and gaps using variable service areas. Transportation, 41(1), 193210. https://doi.org/10.1007/s11116-013-9508-z

Ewing, R., \& Cervero, R. (2010). Travel and the Built Environment. Journal of the American Planning Association, 76(3), 265-294. hitps://doi.org/10.1080/01944361003766766

Ferrocarriles de la Junta de Andalucía. (2008). Modelización de la movilidad actual y futura en el área metropolitana de Granada tras la puesta en servicio de la red de metro ligero (No. Expediente T-MG6109/PEG0). Sevilla.

García-Palomares, J. C., Gutiérrez, J., \& Cardozo, O. D. (2013). Walking Accessibility to Public Transport: An Analysis Based on Microdata and GIS. Environment and Planning B: Planning and Design, 40(6), 1087-1102. https://doi.org/10.1068/b39008

Gehl, J. (1971). Life between buildings: using public space. Danish Architectural Press.

Guerra, E., Cervero, R., \& Tischer, D. (2011). The Half-Mile Circle: Does It Best Represent Transit Station Catchments? (UCB-ITS-VWP-2011-5). Berkeley.

Hass-Klau, C., \& Crampton, G. (2002). Future of urban transport. Learning from success and weakness: light rail (E. and T. Planning, Ed.). Bergische Universität Gesamthochschule Wuppertal.

Hernández, D., \& Witter, R. (2015). Perceived vs. actual distance to transit in Santiago, Chile. Journal of Public Transportation, 18(4). https://doi.org/10.5038/2375-0901.18.4.2 
Hess, D. B. (2012). Walking to the bus: Perceived versus actual walking distance to bus stops for older adults. Transportation, 39(2), 247-266. https://doi.org/10.1007/s11116-011-9341-1

Hess, D. B., \& Almeida, T. M. (2007). Impact of proximity to light rail rapid transit on station-area property values in Buffalo, New York. Urban Studies, 44, 5-6, 10411068. https://doi.org/10.1080/00420980701256005

Jacobs, A. B. (1993). Great Streets. Mit Press.

Jacobson, J., \& Forsyth, A. (2008). Seven American TODs: Good Practices for Urban Design in Transit-Oriented Development Projects. Journal of Transport and Land Use, 1(2), 5188 https://doi.org/10.5198/jtlu.v1i2.67

Kim, S. (2011). Assessing mobility in an aging society: Personal and built environment factors associated with older people's subjective transportation deficiency in the US. Transportation Research Part F: Traffic Psychology and Behaviour, 14(5). https://doi.org/10.1016/j.trf.2011.04.011

Koushki, P. A. (1988). Walking characteristics in Central Riydah, Saudi Arabia. Journal of Transportation Engineering, 114(6). https://doi.org/10.1061/(ASCE)0733-947X(1988)114:6(735) Kuby, M., Barranda, A., \& Upchurch, C. (2004). Factors influencing light-rail station boardings in the United States. Transportation Research Part A: Policy and Practice, 38(3), 223247. https://doi.org/10.1016/j.tra.2003.10.006

Larsen, J., \& El-Geneidy, A. (2010). Beyond the quarter mile: Re-examining travel distances by active transportation. Canadian Journal of Urban Research, 19(1, suppl.), 70-88.

Litman, T. (2016). Accessibility for Transportation Planning Evaluating Accessibility for Transportation Planning. Victoria, Canada. Retrieved from http://vtpi.org/access.pdf

Mitchell, L., Burton, E., Raman, S., Blackman, T., Jenks, M., \& Williams, K. (2003). Making the outside world dementia-friendly: design issues and considerations. Environment And Planning BPlanning \& Design, 30(4), 605-632. https://doi.org/10.1068/b29100

Moniruzzaman, M., Páez, A., Paez, A., \& Páez, A. (2012). A model-based approach to select case sites for walkability audits. Health \& Place, 18(6), 13231334. https://doi.org/10.1016/j.healthplace.2012.09.013

Morency, C., Trepanier, M., \& Demers, M. (2011). Walking to transit: An unexpected source of physical activity. Transport Policy, 18(6), 800-

806. https://doi.org/10.1016/j.tranpol.2011.03.010 
Niemeijer, D., \& de Groot, R. S. (2008). A conceptual framework for selecting environmental indicator sets. Ecological Indicators, 8(1), 14-

\section{5. http://dx.doi.org/10.1016/j.ecolind.2006.11.012}

O'Sullivan, S., \& Morrall, J. (1996). Walking Distances to and from Light-Rail Transit Stations. Transportation Research Record: Journal of the Transportation Research Board, 1538(1), 1926. https://doi.org/10.3141/1538-03

Olszewski, P., \& Wibowo, S. (2005). Using Equivalent Walking Distance to Assess Pedestrian Accessibility to Transit Stations in Singapore. Transportation Research Record: Journal of the Transportation Research Board, 1927, 38-45. https://doi.org/10.3141/1927-05

Park, S., Deakin, E., \& Jang, K. (2013). Can Good Walkability Expand the Size of Transit-Oriented Developments? Transportation Research Record: Journal of the Transportation Research Board, 2519(1), 193-210. https://doi.org/10.3141/2519-17

Pivo, G., \& Fisher, J. D. (2011). The Walkability Premium in Commercial Real Estate Investments. Real Estate Economics, 39(2), 185-219. https://doi.org/10.1111/j.1540-6229.2010.00296.x Pozueta-Echavarri, J., Lamiquiz-Dauden, F., \& Porto Schettino, M. (2009). La ciudad paseable. Centro de Estudios y Experimentación de Obras Públicas.

Seneviratne, P. N. (1985). Acceptable Walking Distances in Central Areas. Journal of Transportation Engineering, 117(4), 365-376. https://doi.org/10.1061/(ASCE)0733947X(1985)111:4(365)

Soria-Lara, J. A. (2011). Modelo de umbrales para la evaluación ambiental de la movilidad urbana. Departamento de Urbanística y Ordenación del Territorio. Granada: Universidad de Granada, Granada. Retrieved from http://www.tdx.cat/handle/10803/80693

Soria-Lara, J. A., Valenzuela-Montes, L. M., \& Pinho, P. (2015). Using 'Mobility Environments' in Practice: Lessons from a Metropolitan Transit Corridor in Spain. Journal of Environmental Policy \& Planning, 17(5), 553-572. https://doi.org/10.1080/1523908X.2014.991779

Talavera-Garcia, R., \& Soria-Lara, J. A. (2015). Q-PLOS, developing an alternative walking index. A method based on urban design quality. Cities, 45, 717. https://doi.org/10.1016/j.cities.2015.03.003

Talavera-Garcia, R., Soria-Lara, J. A., \& Valenzuela-Montes, L. M. (2014). La calidad peatonal como método para evaluar entornos de movilidad urbana. Documents d'Anàlisi Geogràfica, 60(1), 161-187. 
Talavera-Garcia, R., \& Valenzuela-Montes, L. M. (n. d.). Análisis conceptual de la distancia peatonal al transporte público: hacia un enfoque más integrador. ACE Arquitectura, Ciudad y Entorno.

The City of Calgary. (2004). Transit Oriented Development Policy Guidelines. Calgary. Retrieved from https://www.idu.gov.co/documents/20181/1005391/Approved+TODPG+041206.pdf/d d7c6a24-0076-4da1-a6a7-88eaab33b8a3

Valenzuela-Montes, L. M., \& Talavera-García, R. (2015). Entornos de movilidad peatonal: enfoques, factores y condicionantes. Revista EURE - Revista de Estudios Urbano Regionales, 41(123). Retrieved from http://www.eure.cl/index.php/eure/article/view/710/810

Valenzuela Montes, L. M., Soria Lara, J. A., \& Talavera Garcia, R. (2011). Hacia la integración de los planes y proyectos andaluces de movilidad metropolitana. Scripta Nova, 15(349). Retrieved from http://www.ub.edu/geocrit/sn/sn-349.htm

Van de Coevering, P., \& Schwanen, T. (2006). Re-evaluating the impact of urban form on travel patterns in Europe and North-America. Transport Policy, 13(3), 229239. https://doi.org/10.1016/J.TRANPOL.2005.10.001

Venturi, R., Brown, D. S., \& Izenour, S. (1977). Learning from Las Vegas: the forgotten symbolism of architectural form (Revised ed). MIT Press.

Vuchic, V. R. (2005). Urban transit: operations, planning, and economics. John Wiley \& Sons.

Wright, L., \& Hook, W. (2010). Guía de Planificación de Sistemas BRT. New York: Institute for Transportation and Development Policy.

Zacharias, J. (2001). Pedestrian Behavior Pedestrian Behavior and Perception in Urban Walking Environments. Journal of Planning Literature, 16(1), 3-18.

https://doi.org/10.1177/08854120122093249 